\title{
Marine Umweltforschung
}

\section{Ocean Sampling Day: Genomische Schatzsuche im Meer}

\author{
ANNA KLINDWORTH ${ }^{1,2}$, JULIA SCHNETZER ${ }^{1,2}$, IVAYLO KOSTADINOV ${ }^{1}$, RENZO KOTTMANN ${ }^{2}$, FRANK OLIVER GLÖCKNER FR $^{2}$ \\ ${ }^{1}$ JACOBS UNIVERSITY BREMEN \\ ${ }^{2}$ MAX-PLANCK-INSTITUT FÜR MARINE MIKROBIOLOGIE, BREMEN
}

$10.1007 / \mathrm{s} 12268-014-0448-8$

(C) Springer-Verlag 2014

\section{Bedeutung der Mikroorganismen im Ökosystem Meer}

Mikroorganismen bilden einen wichtigen Bestandteil des marinen Planktons. Bereits in einem Tropfen Meerwasser befinden sich über eine Million Mikroorganismen. Als älteste Lebensform der Erde haben sie in den letzten vier Milliarden Jahren sämtliche ökologischen Nischen der Weltmeere besiedelt. Durch ihre große Vielfalt sind sie an allen biologischen Stoffkreisläufen beteiligt und beeinflussen somit direkt das Leben im Meer und an Land. Das Phytoplankton, zu denen auch Cyanobakterien zählen, ist maßgeblich an der Sauerstoffproduktion und Kohlendioxidverwertung auf der Erde beteiligt [1, 2]. Man geht davon aus, dass Phytoplankton für die Produktion von über 50 Prozent des atmosphärischen Sauerstoffs verantwortlich ist, obwohl es nur knapp ein Prozent der weltweiten photosynthetischen Biomasse stellt. Darüber hinaus ist Phytoplankton ein essenzieller Bestandteil der Nahrungskette. Obwohl die zentrale Rolle der marinen Mikroorganismen bekannt ist, steckt unser Wissen über diese, für das bloße Auge unsichtbaren, Meeresbewohner noch in den Kinderschuhen. Der Grund dafür liegt nicht nur in der mikroskopischen Größe der Organismen, sondern darin, dass nur zwischen ein und zehn Prozent von ihnen im Labor kultiviert werden können [3]. Als Revolution erwies sich die Anwendung von kultivierungsunabhängigen Methoden, wie z. B. 16S/18S-rRNA-Gen-Analysen oder die Sequenzierung der gesamten genomischen DNA einer Probe, Metagenomik genannt. Lange Zeit wurden diese Analysen mit dem klassischen Sanger-Sequenzierverfahren durchgeführt, welches jedoch weit entfernt von einem Hochdurchsatzverfahren ist. Noch vor wenigen Jahren konnte man des- halb nur erahnen, welches ungeheure Reservoir an genetischer, physiologischer und synthetischer Vielfalt marine Mikroorganismen besitzen.

Durch den Fortschritt im Bereich Next Generation Sequencing (NGS) können Meeresforscher heute in vergleichsweise kurzer Zeit sehr viele Proben sequenzieren. In den wenigen Jahren seit der Einführung des NGS haben Meeresforschungseinrichtungen aus Europa bereits enorme Mengen an genetischen und genomischen Daten aus dem Meer gewonnen. Um ein besseres Verständnis des marinen Ökosystems zu generieren und dieses Wissen für neue biotechnologische Produkte zu nutzen, müssen diese Daten nun gemeinsam mit einer Vielzahl von Umweltparametern analysiert werden. Während vor wenigen Jahren noch die Datengenerierung der limitierende Faktor war, fehlen heute geeignete bioinformatische Infrastrukturen und Analysesoftware um diese großen multidimensionalen Datensätze auszuwerten.

\section{Interdisziplinarität und Integration: das Micro B3-Projekt}

Im Januar 2012 startete das internationale The Ocean of Tomorrow-EU-Projekt Micro B3 (Marine Mikrobielle Biodiversität, Bioinformatik, Biotechnologie). Prof. Dr. Frank Oliver Glöckner von der Jacobs University und dem Max-Planck-Institut für Marine Mikrobiologie in Bremen koordiniert das Konsortium aus 32 akademischen und industriellen Partnern, das sich aus neun interdisziplinären Expertenteams aus den Bereichen Bioinformatik, Informatik, Biologie, Ökologie, Ozeanografie, Bioprospektion, Biotechnologie, Ethik und Recht zusammensetzt.

Micro B3 hat das Ziel, die großen Mengen an Sequenz- und kontextuellen Daten - auch Metadaten genannt - aus dem Meer für Forscher und Endnutzer verfügbar zu machen sowie die rechtlichen Rahmenbedingungen für die Nutzung mariner genetischer Ressourcen zu entwickeln. Dabei gilt es, biotechnologisch relevante Gensequenzen zu

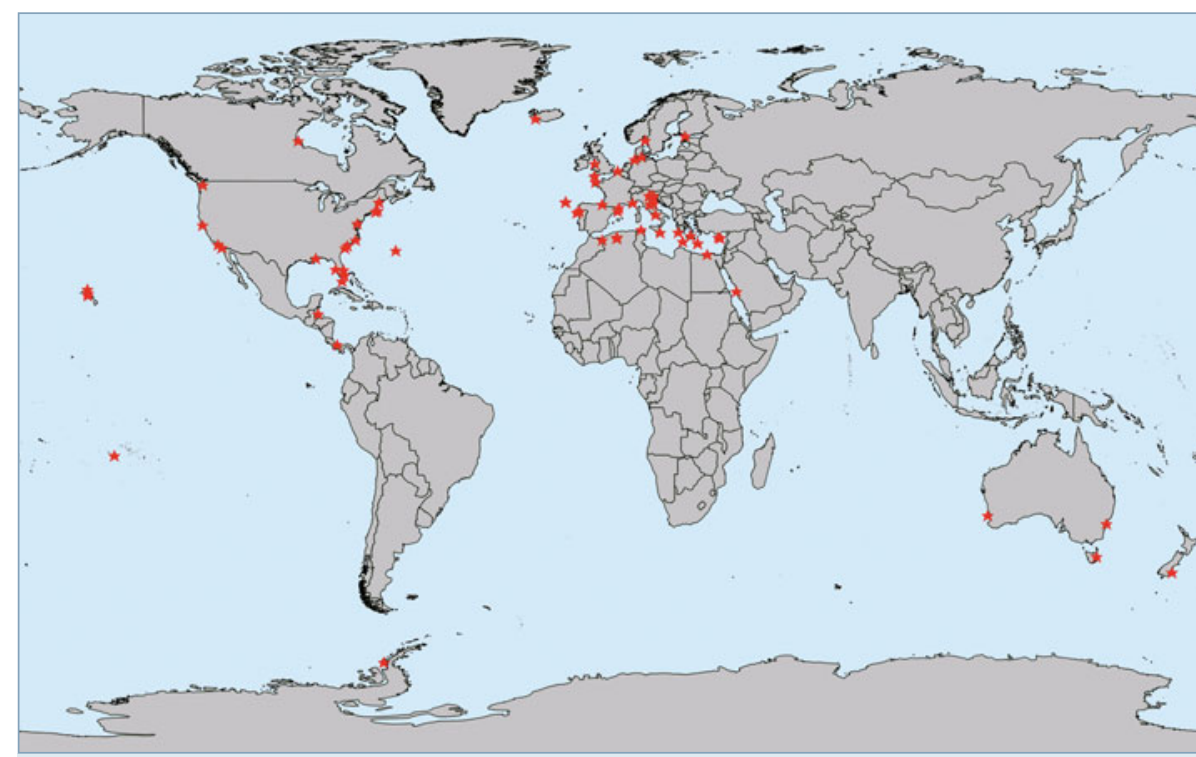

A Abb. 1: Teilnehmer des Ocean Sampling Day (markiert durch rote Sterne; Stand: Januar 2014). 


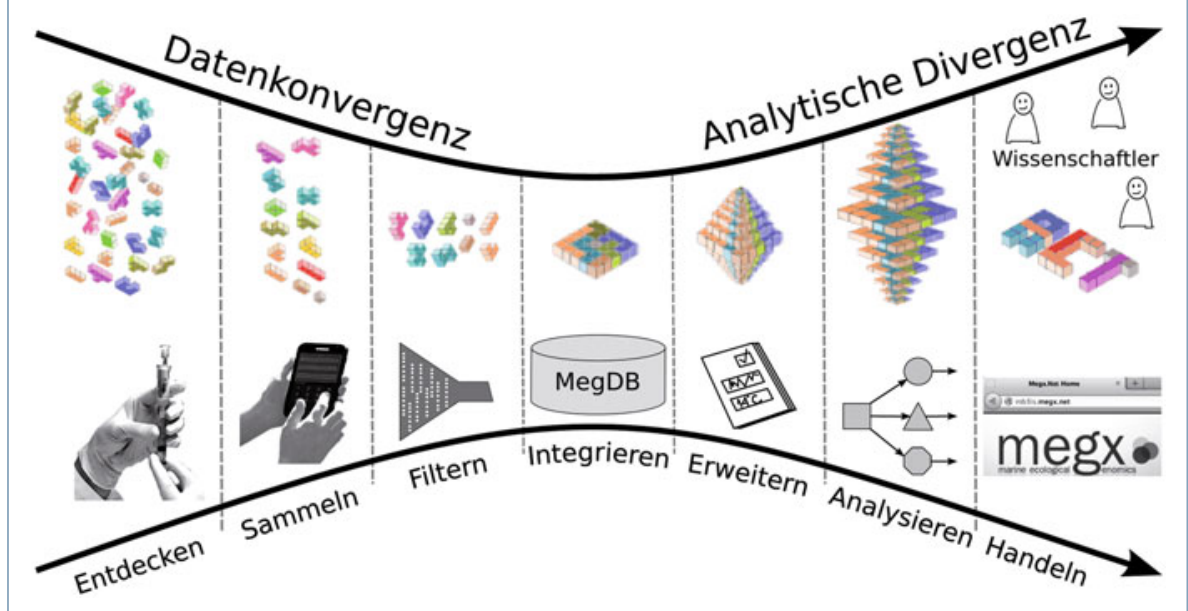

$\Delta$ Abb. 2: Datenfluss innerhalb des Micro B3-Informationssystem. Für die Erhebung der Daten des Ocean Sampling Day werden Endnutzer-Tools entwickelt: Probenahme (Entdecken), Datenakquisition (Sammeln), Pipelines zur Sequenzanalyse (Filtern), Datenbank (Integrieren), CommunitySequenzannotation (Erweitern), ökologische statistische Analysetools (Analysieren) und Websites für die Visualisierung von großen, multidimensionalen Datensätzen (Handeln). Adaptiert von Martin Fowler (http://martinfowler.com/articles/bigData/\#analytics-workflow).

identifizieren und Experimente zu entwerfen, mit denen die Funktion unbekannter Gensequenzen geklärt werden kann. Darüber hinaus werden Musterverträge juristisch ausgearbeitet, die den Zugang und Austausch der genomischen Ressourcen auf internationaler Ebene erleichtern und vereinheitlichen.

\section{Der Ocean Sampling Day}

Das Schlüsselereignis des Micro B3-Projektes ist der Ocean Sampling Day (OSD), der am Tag der Sommersonnenwende, dem 21. Juni 2014, stattfinden wird. An diesem Tag werden Wissenschaftler weltweit nach standardisierten Protokollen Wasserproben aus dem Meer entnehmen, um mikrobielle Diversität und Funktionen zu analysieren und miteinander zu vergleichen. Bisher konnten nahezu 100 Forschungspartner aus den unterschiedlichsten Regionen der Welt für diese Kampagne gewonnen werden. Diese reichen von Europa und Nordamerika bis hin nach Afrika, Australien, Asien und zur Antarktis (Abb. 1).

Die wissenschaftlichen Kernaspekte des OSDs sind vergleichende Diversitäts- und Funktionsanalysen, basierend auf den 16S/18S-rRNA-Genen und Metagenomen. Hierbei wird großer Wert auf die Erhebung von möglichst umfassenden georeferenzierten Umweltdaten gelegt. Diese kontextuellen Daten sind essenziell für die statistische Auswertung und Modellierung des marinen Ökosystems und für die Beantwortung von ökologischen Fragen. Dazu zählen vermeintlich einfache Fragen wie die Ausprägung von Diversität und die genomischen Funktionen von Bakteriengemeinschaften in Abhängigkeit von Umweltfaktoren. Dies ist insbesondere unter dem Einfluss wechselnder chemischer und physikalischer Parameter, wie der globalen Erwärmung, von Bedeutung. Es werden aber auch angewandte Fragen bearbeitet, die darauf abzielen, das Potenzial mariner Mikroorganismen biotechnologisch zu nutzen.

Um diese Ziele zu verwirklichen, muss die Vergleichbarkeit der Ergebnisse gewährleistet sein. Dies ist keine triviale Aufgabe angesichts der zahlreichen Teilnehmer, die über den ganzen Erdball verteilt sind. Die Probenahme und Metadatenerhebung erfolgt daher nach klar definierten Arbeitsschritten und standardisierten Protokollen. Diese wurden in enger Zusammenarbeit mit dem Genomic Standards Consortium (GSC) [4] erstellt. Als Ausgangspunkt dienen hier die Mindestanforderungen der MIXS(Minimum Information about any (x) Sequence)-Spezifikation [5] sowie die Erfahrungen des Earth Microbiome Project (EMP) [6]. Die erarbeiteten OSDStandards und Protokolle sind im Ocean Sampling Day Handbook zusammengefasst (www.microb3.eu/sites/default/files/delive rables/MB3_D4_3_PU.pdf).

Zusätzlich gewährleistet das Micro B3-ABSModel Agreement (Micro B3 Agreement on Access to Marine Microorganisms and Benefit Sharing) den länderübergreifenden Zugang zu marinen genetischen Ressourcen und einen gerechten Vorteilsausgleich. Einzigartig ist auch die Kooperation zwischen Micro B3 und dem Smithsonian-NMNH-Bioarchiv (www.mnh.si.edu/rc/biorepository) in den USA, die es ermöglicht, die Biomasse von bis zu 10.000 OSD-Proben für zunächst fünf Jahre zu lagern. Die Tatsache, dass es möglich sein wird, alle OSD-Proben in einigen Jahren mit den dann aktuellen Technologien reanalysieren zu können, bietet ungeahnte Möglichkeiten und ist in dieser Form einmalig.

\section{Das Micro B3-Informationssystem (Micro B3-IS)}

Der OSD-Datensatz ist der größte standardisierte marine Datensatz, der je an einem einzigen Tag zusammengetragen wird und liefert eine möglichst umfassende mikrobiologische Momentaufnahme der Weltmeere. Um diese enorme Menge an Daten verwalten und analysieren zu können, wird das Micro B3Informationssystem (Micro B3-IS) entwickelt, das die zentrale bioinformatische toolbox des Projektes darstellt (Abb. 2). Es basiert auf dem megx.net-System (Marine Ecological GenomiX) [7] und integriert Informationen aus Umweltdatenbanken wie PANGAEA, SeaDataNet, EurOBIS sowie Sequenzdaten aus dem European Nucleotide Archive (EMBLEBI/ENA) in ein zentrales Datenmanagement und Informationssystem (Abb. 3). Das Micro B3-IS ermöglicht somit eine flexible, transparente und benutzerfreundliche Integration, Analyse und Visualisierung von großen, multidimensionalen Datensätzen aus laufenden Biodiversitätsstudien und Langzeitbeobachtungen. Dadurch entsteht ein integriertes Wissen, das neue Perspektiven für die Modellierung und Ökosystemforschung im Meer eröffnet. Dennoch ist klar, dass für ein tief greifendes Verständnis der Reaktionen von marinen Mikroorganismen auf den Klimawandel der Ocean Sampling Day nur den Beginn einer kontinuierlichen, globalen genomischen Beprobung markieren kann [8].

\section{OSD-Netzwerk und Citizen Science}

Das Micro B3-Projekt und der Ocean Sampling Day helfen Wissenschaftlern, sich weltweit besser zu vernetzen. Wir möchten jedoch noch einen Schritt weiter gehen und ermuntern interessierte Bürgerinnen und Bürger, im Rahmen unseres OSD-Citizen-Science-Projektes (www.my-osd.org) die Wissenschaftler tatkräftig bei der Erhebung von Umweltdaten zu unterstützen. Dabei können sie wichtige Parameter wie Luft- und Wassertemperatur mit relativ einfachen Mitteln messen und im Anschluss mit der neu entwickelten OSD-App für Smartphones oder direkt über eine Web- 


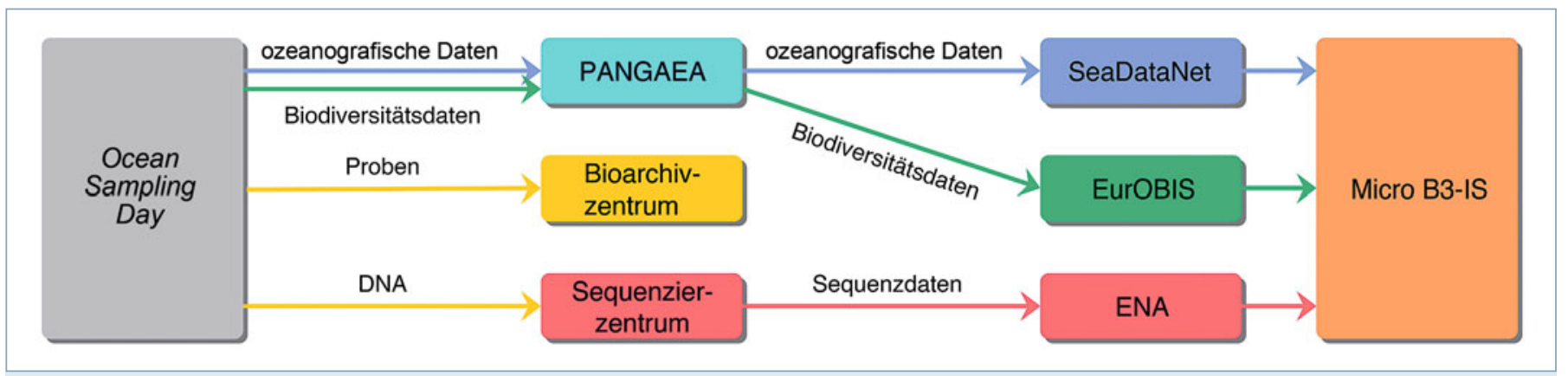

A Abb. 3: Datenfluss von den Probenahmen bis zur Datenintegration und Auswertung beim Micro B3-IS-Projekt. Informationen aus Umweltdatenbanken wie PANGAEA, SeaDataNet, EurOBIS sowie Sequenzdaten aus dem European Nucleotide Archive (EMBL-EBI/ENA) werden in ein zentrales Datenmanagement und Informationssystem integriert.

seite zum Micro B3-Informationssystem hochladen.

Alle Daten werden innerhalb des Micro B3-

IS gesammelt und stehen interessierten Wissenschaftlern und Bürgern für weitere Analysen zur Verfügung. Bürger haben somit die Möglichkeit, aktiv an der Wissenschaft teilzunehmen und die Meeres- und Klimaforschung zu unterstützen.

Weitere Informationen über das Projekt und der Teilnahme am Ocean Sampling Day finden Sie unter www.oceansamplingday.org (für Wissenschaftler) und www.my-osd.org (für Citizen Scientists).

\section{Danksagung}

Wir möchten uns hiermit bei allen Micro B3Partnern, OSD-Teilnehmern und interessierten Bürgern herzlich für ihre Unterstützung und Mitarbeit bedanken.

\section{Literatur}

[1] Field CB, Behrenfeld MJ, Randerson JT et al. (1998) Primary production of the biosphere: integrating terrestrial and oceanic components. Science 281:237-240 [2] Kirchman DL, Morán XA, Ducklow H (2009) Microbial growth in the polar oceans - role of temperature and potential impact of climate change. Nat Rev Microbiol 7:451-459

[3] Amann RI, Ludwig W, Schleifer KH (1995) Phylogenetic identification and in situ detection of individual microbial cells without cultivation. Microbiol Rev 59:143-169 [4] Yilmaz P, Gilbert JA, Knight R et al. (2011) The genomic standards consortium: bringing standards to life for microbia ecology. ISME J 5:1565-1567

[5] Yilmaz P, Kottmann R, Field D et al. (2011) Minimum information about a marker gene sequence (MIMARKS) and minimum information about any $(\mathrm{x})$ sequence $(\mathrm{MIxS})$ specifications. Nat Biotechnol 29:415-420

[6] Gilbert JA, Meyer F, Jansson J et al. (2010) The Earth Microbiome Project: meeting report of the "1st EMP meeting on sample selection and acquisition" at Argonne National Laboratory October 6th 2010. Stand Genomic Sci 3:249-253 [7] Kottmann R, Kostadinov I, Duhaime MB et al. (2009) Megx.net: integrated database resource for marine ecological genomics. Nucleic Acids Res 38:D391-D395

[8] Davies N, Field D, Genomic Observatories Network (2012) Sequencing data: a genomic network to monitor Earth. Nature 481:145-145

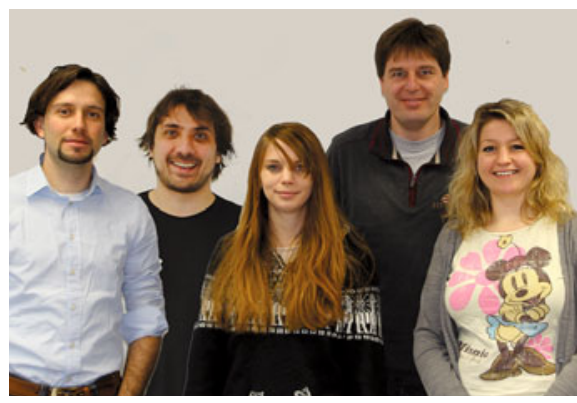

Ivaylo Kostadinov, Renzo Kottmann, Julia Schnetzer, Frank Oliver Glöckner und Anna Klindworth (v. I. n. r.)

Korrespondenzadresse:

Prof. Dr. Frank Oliver Glöckner Jacobs University Bremen gGmbH Campus Ring 1

D-28759 Bremen

Tel.: 0421-200-3167

Fax: 0421-200-3249

f.gloeckner@jacobs-university.de 